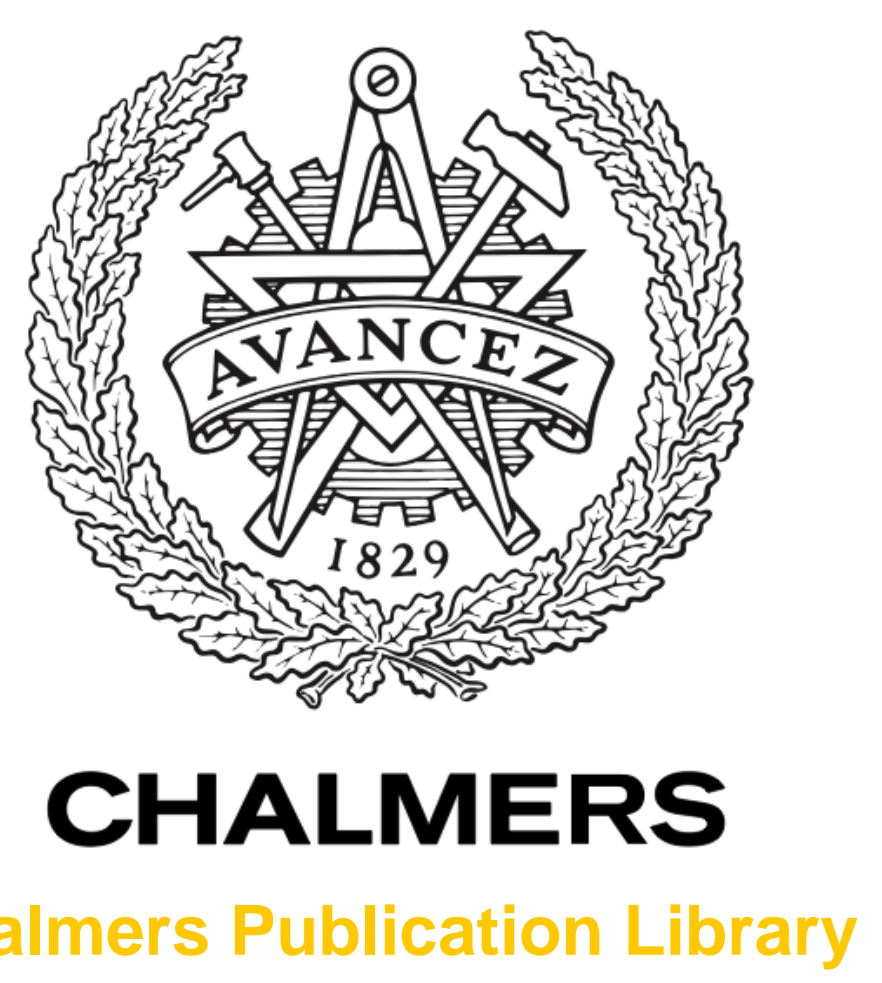

Chalmers Publication Library

\title{
Understanding differences in MAC performance
}

This document has been downloaded from Chalmers Publication Library (CPL). It is the author's version of a work that was accepted for publication in:

\author{
IEEE WoWMoM Workshop on Smart Vehicles: Connectivity Technologies and ITS \\ Applications \\ Citation for the published paper: \\ Gaugel, T. ; Mittag, J. ; Hartenstein, H. (2014) "Understanding differences in MAC \\ performance". IEEE WoWMoM Workshop on Smart Vehicles: Connectivity Technologies \\ and ITS Applications
}

Downloaded from: http://publications.lib.chalmers.se/publication/197582

Notice: Changes introduced as a result of publishing processes such as copy-editing and formatting may not be reflected in this document. For a definitive version of this work, please refer to the published source. Please note that access to the published version might require a subscription. 


\title{
Understanding differences in MAC performance
}

\author{
Tristan Gaugel*, Jens Mittag*, Hannes Hartenstein*, Erik G. Ström ${ }^{\dagger}$ \\ \{tristan.gaugel, jens.mittag, hannes.hartenstein\}@kit.edu, erik.strom@chalmers.se \\ *Institute of Telematics \& Steinbuch Centre for Computing, Karlsruhe Institute of Technology (KIT) \\ ${ }^{\dagger}$ Dept. of Signals and Systems, Chalmers University of Technology
}

\begin{abstract}
The suitability and performance of medium access protocols in vehicular environments is already being investigated over a long period of time. Carrier Sense Multiple Access (CSMA) has been shown to perform sufficiently well in most situations and being able to support safety and efficiency vehicular applications. Recently, Self-organizing Time-Division Multiple Access (STDMA) is being considered as an alternative and has been shown to coordinate the channel slightly better under certain situations. However, when comparing both protocols the precise details of radio and network conditions and parametrization of the protocols are decisive on which protocol takes a slight lead. Consequently, scenarios can be constructed quite easily in which one protocol is superior over the other one. The focus of this work is thus not to absolutely compare both protocols, but rather to understand the strengths and weaknesses of both protocols in certain situations. In particular, we consider i) to which degree hidden nodes influence the coordination ability, ii) how an extended carrier sensing range is beneficial and iii) how temporary fading influences the performance of both MAC protocols. Our results show that while an extended carrier sensing range is only beneficial for CSMA, the existence and severity of fading is far less detrimental for STDMA than for CSMA.
\end{abstract}

\section{INTRODUCTION}

Vehicular communication, by means of Dedicated Short Range Communication (DSRC), strives to improve safety and efficiency on our roads by creating a more extensive awareness between participating vehicles. The periodic exchange of beacons hereby serves as a basis to create a local view on the surrounding on which applications can then decide on, e.g., whether and when to warn a driver. In order to achieve an up-to-date and exhaustive view, an effective medium access scheme is necessary on which vehicle is allowed to transmit at which point of time (and for which period).

In the area of vehicular networks the use of Carrier Sense Multiple Access (CSMA) has been studied in detail over the last decade, it is standardized in the IEEE 802.11p amendment and it has been shown to perform sufficiently well under most conditions (cf. [7]). Self-organizing Time-Division Multiple Access (STDMA), which employs a reservation based scheme, contrary to the random based access scheme of CSMA, is standardized for a similar purpose of exchanging beacons in maritime [4] and aerial [1] settings. In recent work ([9], [10]) it has on the one hand been shown that STDMA is a serious alternative to CSMA in a vehicular setting, but on the other hand it also became obvious that the precise details of, e.g., radio and network conditions are decisive on which protocol takes a slight lead. As a consequence, the focus of our work is not to absolutely compare both protocols, but to understand the conditions and situations in which one protocol has more difficulties, or gains benefits, compared to the other one. This information can furthermore be used by application designers to identify critical communication situations and design their applications with this in mind.

In this paper we analyze how the following conditions influence the performance of STDMA in comparison to CSMA:

1) Existence and ratio of hidden nodes

2) Ratio of the distance at which packets can no longer be decoded successfully (reception range), but the channel can still be sensed as busy (carrier sensing range)

3) Existence and severity of fading conditions, leading to temporary fluctuations of packet receptions

These situations help us to identify the strengths and weaknesses of both protocols in dealing with varying radio conditions and to which degree both protocols are able to handle dynamics, introduced by fading conditions.

The rest of this paper is structured as follows: In Section II we present related work. Section III provides an overview on our simulation methodology and introduces the metrics that are used in the evaluation. In Section IV the results of our wireless network simulation are presented and assessed. Section V concludes the paper with a summary and outlook.

\section{RELATED WORK}

Research on MAC protocols, which coordinate the access on a shared medium, dates back until the 1970s. In the meantime several protocols have been proposed, employing different approaches on how to coordinate the channel access. For a general introduction and survey over different approaches we refer to Gummalla et al. [2], more detailed information concerning MAC protocols for VANETs can be found in Section 7 of [3]. In the following we are only focusing on CSMA and STMA.

CSMA employs a 'listen before talk'-principle where every node first listens on the channel whether a transmission is ongoing before transmitting itself. In case the channel is sensed as busy, the own transmission is postponed for a random backoff. The first work to consider the influence of hidden nodes on CSMA was Tobagi et al. [11] in 1975. Subsequent works then considered the impact of protocol elements or that of technical improvements as well as considered more realistic channel propagation models for evaluating the performance of CSMA. Recent work (cf. [7]) shows that CSMA is able to effectively coordinate the channel access in vehicular networks. 


\begin{tabular}{l|l||l|l}
\hline Scenario, application layer and common MAC settings \\
\hline Number of stations & $177(85$ vehicles/km) \\
Packet size & $400 \mathrm{bytes}$ & \\
Transmission rate\&power & $10 \mathrm{~Hz}, 20 \mathrm{dBm}$ & \\
CS/RX-threshold & $-91 /-88 \mathrm{dBm}$ & \\
\hline \multicolumn{3}{c}{ STDMA based MAC } & CSMA based MAC \\
\hline Frame duration & $1 \mathrm{sec}$ & Slot time & $13 \mu \mathrm{s}$ \\
Min. candidate slots & $1 \mathrm{slot}$ & Contention- & 15 slots \\
Reservation duration & $\mathcal{U}[3,7]$ frames & window size & \\
Selection interval ratio & $20 \%$ & & \\
Guard interval per slot & $6 \mu \mathrm{s}$ & \\
\hline
\end{tabular}

TABLE I

APPLICATION AND MEDIUM ACCESS CONTROL LAYER PARAMETERS USED.

STDMA on the other side employs a reservation based scheme where every node previously announces its transmissions. For this purpose the time is divided in so called slots, constituting a frame of fixed length. For a more detailed description of the protocol and an explanation of protocol elements we refer to Gaugel et al. [10]. The severity of the existence of hidden nodes has been studied by Sjöberg et al. [5], by means of the packet reception probability, and found not to be a major limiting factor. In general, Sjöberg [9] found STDMA to be highly competitive and even partly exceeding the performance of CSMA.

\section{Methodology}

In this chapter first the methodology of our simulation-based evaluation is presented and the metrics are introduced that are used to quantify the performance of CSMA and STDMA. All simulations were performed with NS-3 in version 3.16.

\section{A. Scenario layout and general settings}

In all our scenarios, stationary nodes are positioned on a straight road with a length of 2100 meters. We assumed equally distributed inter-vehicle distances with a vehicle density of 85 vehicles $/ \mathrm{km}$, resulting in a total of 177 vehicles. Every node is configured to transmit 10 packets per second with a packet size of 400 bytes and in combination with a data rate of $6 \mathrm{Mbps}$ this results in an experienced channel load of $10 \%$ per $100 \mathrm{~m}$ carrier sensing range (CS-range). A CS-range of 500 meters consequently leads to experienced $50 \%$ channel congestion or in other words $3 \mathrm{Mbps}$ of transmissions that can be carrier-sensed. We successively start up our nodes with a rate of one node per second and start the evaluation after every node is started up for a total of 180 seconds. While every node is actively participating in the vehicular network (transmitting and receiving) we are only evaluating packets that are transmitted from vehicles positioned in the central 100 meters of the road. This way boundary effects are avoided that result in a significant drop of the experienced channel load at the edge of the scenario. A summary of important settings can be found in Table I.

\section{B. Radio propagation model and MAC-settings}

In total three different radio models were considered in order to point out different characteristics of the MAC protocols and to work out the impact of varying conditions on the performance of the protocols.
1) Fixed range communication model: This model serves as a basis for the further work, since it facilitates interpretation of the achieved results and thus allows to better highlight the causes leading to incoordination or packet drops. The received signal strength at a receiver located $d$ meters away from the transmitter is set to the transmission power if $d \leq$ communication range, otherwise the received power is zero. This ensures that a packet is successfully delivered if i) the distance between transmitter and receiver is less than the configured communication range (CR) and ii) no other node within the $\mathrm{CR}$ of the potential receiver is interfering or, in other words, transmitting a packet with an overlap in time.

2) Power law communication model: This model employs a log distance communication model with a path loss exponent of 1.85 and a reference loss of $59.7 \mathrm{dBm}$ at a distance of $1 \mathrm{~m}$. These parameters arise from measurements performed by Kunisch et al. [6] in vehicular environments. Contrary to the fixed range communication model this model allows to separate between a carrier sensing range and a receiving range, the range at which a signal is strong enough that a potential receiver can sync to the signal. If not otherwise stated we assumed a CS-threshold of $-91 \mathrm{dBm}$ and an RX-threshold of $-88 \mathrm{dBm}$, which equals a CS-range of approx. 600 meters, when only considering deterministic path loss, and a receiving range of approx. 400 meters. In addition, the decrease of signal strength over distance leads to two general differences, compared to the case of fixed range communication: i) packets might still be successfully received even if a packet reception is being interfered, if the resulting signal to interference and noise ratio (SINR) for the current reception is still sufficiently large enough, ii) non successful packet receptions might occur even if the potential receiver is within the deterministic communication range, since packets might be dropped by the packet error rate model(which is the NistErrorRateModel of NS-3 in our case), whose probability depends on the SINR. As a consequence, the results achieved by using this model considers the distance at which an incoordination occurs in more detail, where interferences in close range are generally worse than if the interferer is located far away.

3) Nakagami fading communication model: This model employs fading by a Nakagami distribution [8] on top of the power law path loss model explained previously. The Nakagami m-parameter hereby indicates the severity of fading, where a low value indicates severe fading (e.g. $m=1$ equals Rayleigh fading) and a higher value (e.g. $m=3$ ) represents a reduced fading intensity. Generally speaking, fading leads to temporary signal gains or decreases and hence to an temporary increase or decrease up to which distance a packet can be delivered. This model thus allows us to assess the effect of temporary radio propagation variations on the overall performance that the protocols are able to achieve. ${ }^{1}$

\footnotetext{
${ }^{1}$ Typically, a Nakagami distribution is used to model small-scale fading, i.e., variations that occur several times while a packet is transmitted, whereas slowfading is assumed to be log-normal distributed and not varying in the time of a single packet transmission. For our analysis the specific characteristic of fading is not crucial and we decided to model it as independent and identically distributed large-scale fading by means of a Nakagami distribution.
} 


\section{Metrics}

In order to assess the performance of the MAC protocols under varying conditions and in different situations we are using the following metrics:

Definition 1 (Packet Level Incoordination, PLI): The packet level incoordination, as observed from the perspective of a node $r$ and one of its generated packets $p$, describes the probability that, at least, one node $s, s \neq r$, transmitted a packet $q$ during the transmission period of $p$.

The PLI is a transmitter-based metric which captures how well a MAC protocol is able to prevent simultaneous transmissions which most protocols try to avoid in a first step.

Definition 2 (Packet Delivery Ratio, PDR): The packet delivery ratio, as observed from the perspective of a node $r$, describes the probability that a packet transmitted by node $r$ is successfully received at a potential receiver $s, s \neq r$.

The PDR is a receiver-based metric and implicitly considering factors like i) the interference caused by incoordinated transmissions or ii) the capability of the receiver to e.g. cope with noisy receptions (needed SINR) or being able to switch to a stronger signal at the expense of a weaker signal that is dropped (packet capturing).

How are the metrics connected to each other? In the following we want to discuss which relationship between PLI and PDR can be expected. We are therefore assuming an STDMA based approach, since the concept of time slots simplifies our analysis, the general relationship between PLI and PDR, however, stays the same for CSMA. For this analysis another metric is needed:

Definition 3 (Slot Occupation Distribution, SOD): The slot occupation distribution describes the probability that a transmission slot is used by exactly $i$ nodes, $i=0,1,2,3 \ldots$. When $n_{i}$ represents the number of slots occupied by exactly $i$ transmissions and $n=\sum_{i=0}^{\infty} n_{i}$ is the total number of slots, these probabilities can be calculated as $\frac{n_{i}}{n}$.

For a first analysis we assume a highly idealistic small scale scenario with a fixed range communication model and every node within the communication range of each other. Under these assumptions a transmission is successfully received if and only if one slot is exactly occupied by one station, whereas no packets can be decoded if a slot is used by more than one station. Under these conditions the PLI and PDR can both be derived from the SOD as follows:

$$
\begin{gathered}
P L I=\sum_{i=2}^{\infty}\left(\frac{(i-1) n_{i}}{a}\right) \\
P D R=\frac{n_{1}}{a}
\end{gathered}
$$

where $a$ equals the number of overall transmission attempts, calculated as $a=\sum_{i=1}^{\infty}\left(i n_{i}\right)$. For calculating the $P L I$ we have to sum up the number of nodes experiencing incoordination in a certain time slot (which is every node except the first one transmitting in this time slot) and divide it by the overall number of transmission attempts. For calculating the PDR only the slots have to be summed up with exactly one transmission taking place and divided by the overall transmission attempts.
Looking at Equation (1) and Equation (2) the relation can be stated as:

$$
P D R=1-c * P L I
$$

where the parameter $c$ depends on the slot occupation distribution. Note: For another definition of the PLI, as $P L I=\frac{a-n_{1}}{a}$, the relation can be simplified as $P D R=1-P L I$.

In a more realistic scenario with hidden terminals and varying characteristics of the radio channel the PLI can still be calculated by using the slot occupation statistics, however, calculating the PDR in the same way is no longer possible. The reason therefore is that slot reusing might become a successful option or, in other words, packets transmitted by multiple nodes within the same time slot do not necessarily prohibit successful receptions of these packets at distinct nodes. In order to determine whether a packet is successfully received, plenty of factors have to be considered, like on the one hand the receiver characteristic that determines the packet error rate or the ability to perform packet capturing. On the other hand the radio characteristic also plays an important role in determining the interference caused by simultaneous transmissions at a receiver. All these aspects are hard to evaluate in an analytical way and we are thus contrasting and quantitatively measuring the PLI and PDR in Section IV by means of a simulation based study.

\section{RESUlts}

In this chapter the results of our simulation based study are presented that give insights on which situations are affecting the ability to successfully coordinate the channel to which degree. All the results presented are average values, based on five runs with different seeds for the random number generator. The resulting variance with five runs is already too low to be visible in the presented plots.

\section{A. Impact of ratio of hidden terminals}

In order to determine how the coordination capability of both protocols is solely affected by the existence of hidden terminals, without having to consider varying ranges at which a nodes becomes a hidden terminal or the effect of packet error rate models, we employed a fixed range propagation loss model in a first step. The communication range is set to 500 meters, meaning that every node within this range can successfully receive a transmission if no other node within a distance of 500 meters transmits a packet itself.

In Figure 1 the resulting PLI for a scenario with a theoretical channel load of $50 \%$ is depicted, depending on the ratio of hidden nodes to overall nodes and the MAC protocol being used. The number of vehicles that are within communication range of an evaluation node hereby stays the same in all setups and corresponds to $50 \%$ channel load. A hidden node ratio of $1 / 2$ denotes that the vehicle density stays the same over the whole scenario, whereas the vehicle density is reduced to half at distances larger 500 meters for a hidden node ratio of $1 / 3$. In other words: From the perspective of a single node, a hidden node ratio of $1 / 3$ denotes that out of all vehicles that can interfere my transmissions $1 / 3$ is hidden to me. 


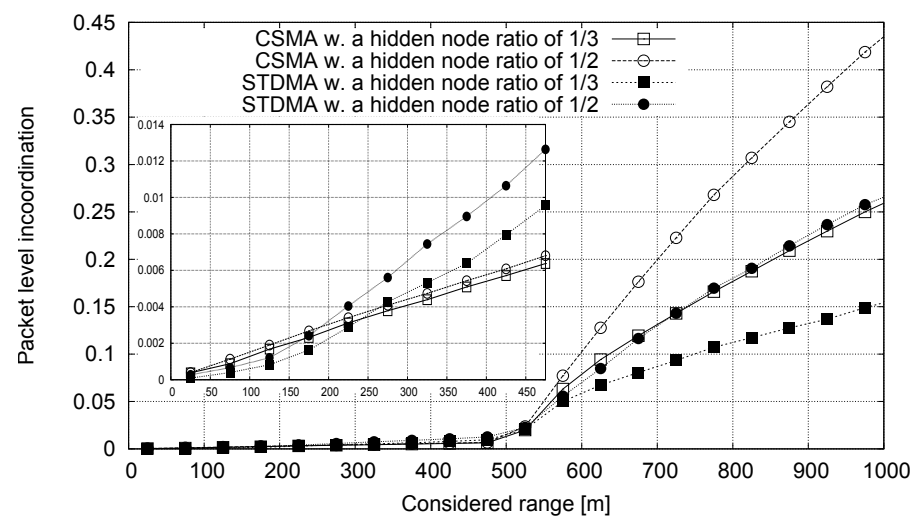

Fig. 1. PLI with an estimated channel load of $50 \%$ using a fixed range propagation loss model with a range of $500 \mathrm{~m}$ for a considered range up to $1000 \mathrm{~m}$ and zoomed in to illustrate the range up to $500 \mathrm{~m}$ in more detail.

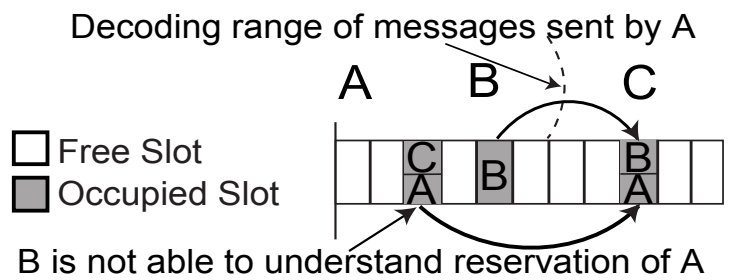

Fig. 2. Illustration of how a hidden node (Node C) can lead to incoordination between two nodes that are within each others communication range (Node A and B) with STDMA

Three general observations can be made in Figure 1. First, the overall PLI up to a distance of 500 meters is quite low for both protocols, concluding that both protocols are well capable of coordinating the channel within the communication range, even with the existence of hidden nodes. Second, the PLI drastically increases at distances larger than 500 meters, since CSMA is no longer able to carrier sense ongoing transmissions and STDMA is no longer able to extract reservation information originating from distances larger than 500 meters. Third, the more hidden nodes are present the higher the PLI gets for both protocols. For a study on how the PLI of CSMA and STDMA are affected by varying channel load we refer to our previous publication [10].

Furthermore, when comparing the PLI for STDMA and CSMA in more detail, following differences are observable:

i) The PLI for CSMA increases drastically faster compared to STDMA at distances larger than $500 \mathrm{~m}$. The reason therefore is that STDMA uses a slotted approach, where transmission times between different nodes are synchronized. An incoordinated transmission can thus only interfere with other transmissions taking place in the same time slot when using STDMA (full overlap), where with a non synchronized approach like CSMA an incoordinated transmission can interfere with transmissions starting any time from the first to last bit (partly overlap).

ii) In close range the PLI for CSMA linearly increases over distance as incoordination with CSMA within the communication range occurs if multiple nodes decide to transmit at the same point of time (e.g. by an equal backoff timeout, cf. [7]). iii) In close range the PLI for STDMA increases with an exponential trend. In order to be able to explain this behavior we first have to identify how hidden nodes can lead to incoordination between two nodes when using STDMA. In our previous publication [10] we showed that with the absence of hidden nodes and under the load that is considered here, there is rarely the chance of any incoordination. Figure 2 now depicts a possibility of how two nodes that are within each others communication range can end up in an incoordinated transmission because of the existence of a hidden node: Node $\mathrm{B}$, which is within the communication range of node $\mathrm{A}$ misses its reservation information, since Node $\mathrm{C}$ interferes. Node B is then by chance selecting the same next slot to transmit in, leading to incoordination between Node A and B. Since packet collisions can be of contagious nature (cf. [10]) this can then again lead to new incoordinations between nodes, that are within each others communication range. Contrary to CSMA, the probability of incoordinated transmissions increases with an increase of hidden nodes, since more nodes are able to interfere with reservation information exchanged within the communication range. This can be seen by comparing the PLI for a hidden node ratio of $1 / 3$ vs. $1 / 2$. Additionally, as the distance increases, the number of vehicles that are outside of the communication range (thus generally not coordinated), but still within interference range of a potential receiver increases, leading to an even greater increase of incoordination.

Consequently, for contrasting the PLI of CSMA and STDMA, Figure 1 can be divided into three different regions:

1) Up to a distance of $\approx 200$ meters (for the given scenario) there is a higher probability of simultaneous packet transmissions by CSMA than hidden nodes interfering/destroying reservations when using STDMA.

2 ) In the range from $\approx 200$ meters to $\approx 550$ meters the effect of hidden nodes interfering with reservation information leads to more PLI for STDMA than that of additional nodes starting their transmissions at the same point of time when using CSMA.

3) For larger distances (depicting the probability of hidden nodes being coordinated) the visible difference in PLI is due to the effect of slotted vs. non slotted approach, favoring STDMA over CSMA.

Figure 3 visualizes the resulting PDR in combination with the PLI for the scenario with a hidden node ratio of $1 / 2$. Keep in mind that the PDR at some distance $d$ is not only influenced by all incoordinations occurring up to distance $d$, but by incoordinations occurring up to a distance of $d+$ communication range, as is depicted in Figure 3.

For the first $25 \mathrm{~m}$ the PDR of CSMA is slightly better by $1 \%$, resulting from the marginal PLI benefit of CSMA within the communication range (cf. Figure 1). For larger distances the difference of slotted vs. non slotted approach is more decisive. The PDR does not reach $100 \%$ even in close range, since any interference within the first $500 \mathrm{~m}$ hinders a successful packet reception. At distances above 500 meters no single packet can be decoded, as is expected with a fixed range communication model. 


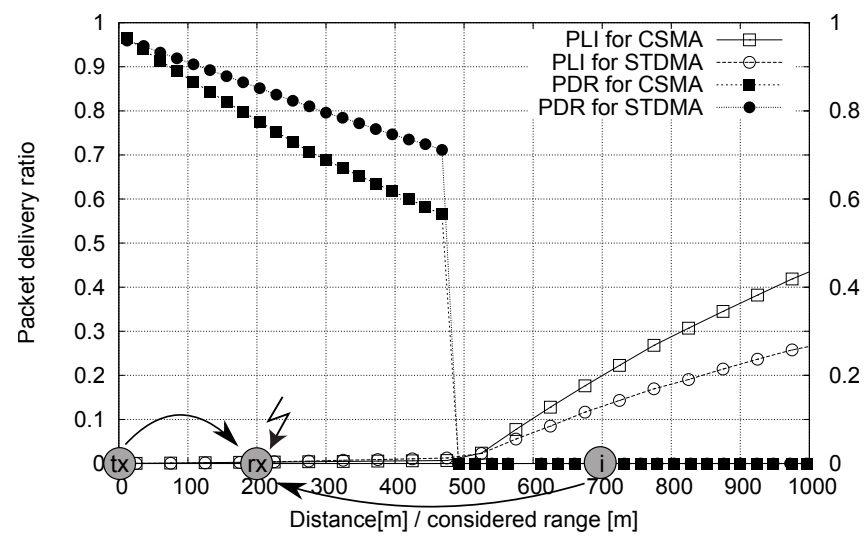

Fig. 3. PLI and PDR for a scenario with a theoretical channel load of $50 \%$, a hidden node ratio of $1 / 2$ and a fixed communication range model with $500 \mathrm{~m}$. The PDR of node $t x$ at a distance of 200 meters (represented by node $r x$ ) is influenced by all incoordination, occurring up to node $i$ at $700 \mathrm{~m}$, which can eventually lead to packet collision at node $r x$.

In a next step we changed the communication model to the power law model (still with CS-threshold $=$ RX-threshold) in order to quantify the impact of a different path loss characteristic. Due to space restrictions we are not able to present the results in detail here, however, they are implicitly included in Figure 4 for a CS-Range of $400 \mathrm{~m}$ and in the resulting PDR for STDMA in Figure 5.

\section{B. Impact of varying CS-ranges}

Our last study revealed that as long as both protocols experience the same amount of hidden nodes, the slotted approach of STDMA results in better coordinating abilities. However, CSMA's coordination mechanism primarily relies on being able to sense the channel, on the contrary STDMA primarily relies on being able to decode packets in order to extract reservation information. Consequently, the following questions are addressed next i) how does the PLI changes, depending on the relation between carrier sensing and decoding range or in other words how much benefit can both protocols gain by an extended carrier sensing range and ii) how does this influence the achievable PDR? For this study we employed the power law fading model. The threshold up to which a receiver is able to sync to a signal is set to $-88 \mathrm{dBm}$, which equals a distance of $408 \mathrm{~m}$. Please keep in mind that even when a receiver is able to sync to a packet it does not necessarily imply a successful reception, since the packet might still be dropped by the packet error rate model, due to non sufficient SINR. For this study the CS-range is varied from $400 \mathrm{~m}$ to $1000 \mathrm{~m}$.

Figure 4 visualizes the PLI for varying CS-ranges for CSMA and STDMA. As can be seen CSMA greatly benefits from an increase of the CS-range whereas the shape of the curve stays comparable to the one observed in Figure 1. The increase of the PLI in close range can be explained by the fact that with a larger CS-range the experienced channel load increases. An increase of channel load leads to more competition for the remaining free channel times, leading to a higher probability of simultaneous backoff timeouts. STDMA can in general also make use of sensing the channel as busy

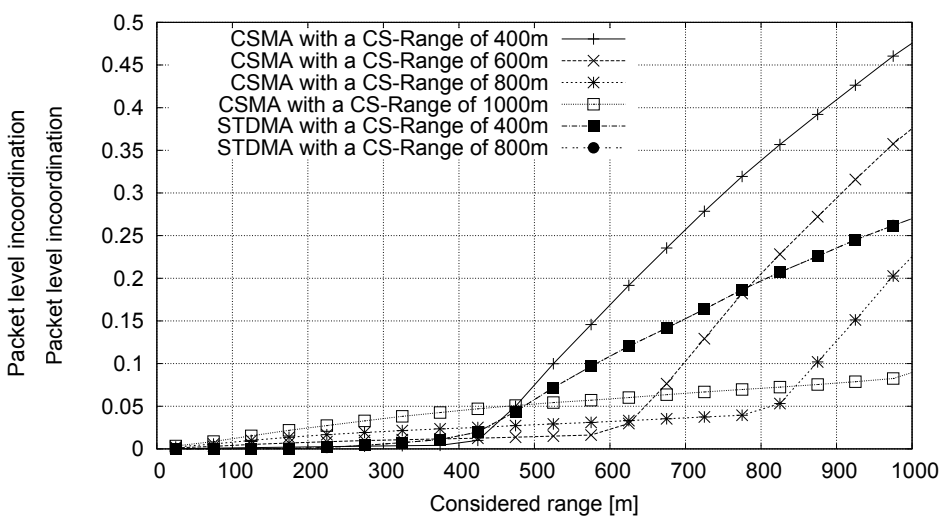

Fig. 4. PLI for various carrier sensing ranges with a maximum decoding range of $\approx 400 \mathrm{~m}$ and a density of 85 vehicles $/ \mathrm{km}$

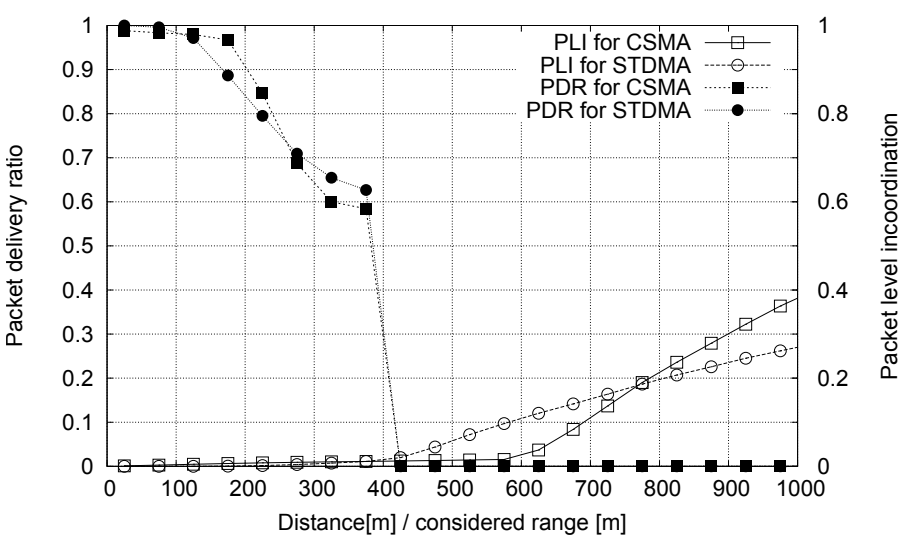

Fig. 5. PLI and PDR for a CS-Range of $\approx 600 \mathrm{~m}(-91 \mathrm{dBm})$, a maximum decoding range of $\approx 400 \mathrm{~m}(-88 \mathrm{dBm})$ and a vehicle density of $85 \mathrm{veh} / \mathrm{km}$

by blocking the same slot in the next frame, but in our setup no difference is observable when extending the CSrange. However, in close range (up to $\approx 200 \mathrm{~m}$ ) the PLI drops significantly, compared to the results achieved with the fixed range communication model. For an explanation consider the scenario depicted in Figure 2 where the distance between node $\mathrm{A}$ and $\mathrm{B}$ is rather small compared to the distance between $\mathrm{B}$ and C. Consequently, the signal strength of the packet received by node $\mathrm{A}$ is considerably stronger than the one received by node $B$ and the resulting SINR might still be sufficient to decode the packet from node A and being able to extract its reservation information.

In Figure 5 the PLI for a CS-threshold of $-91 \mathrm{dBm}$ $(\approx 600 \mathrm{~m})$ and an RX-threshold of $-88 \mathrm{dBm}(\approx 400 \mathrm{~m})$ is depicted in combination with the resulting PDR for CSMA and STDMA. The higher PDR for STDMA in ranges up to approx. $100 \mathrm{~m}$ is due to constantly lower PLI in ranges up to $400 \mathrm{~m}$ and incoordination occurring at larger distances only having minor effect on the SINR. CSMA has its strengths (regarding the PDR) in the range from $100 \mathrm{~m}$ to $250 \mathrm{~m}$, since it benefits from an extended CS-range by being able to coordinate the channel up to larger distances. Due to its slotted approach the PLI changes again in favor of STDMA at distances larger than $750 \mathrm{~m}$, resulting in higher PDRs for distances larger than 250 meters (cf. Section IV-A for explanation). 


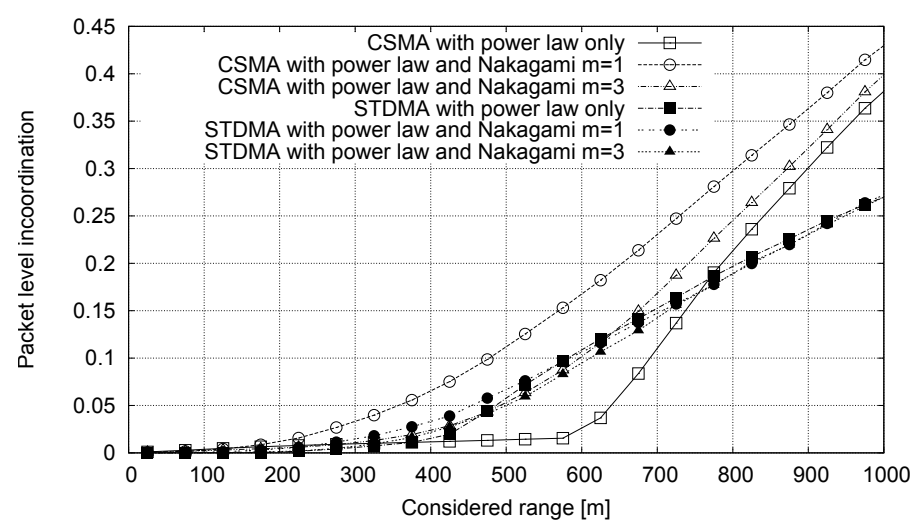

Fig. 6. Impact of fading with varying intensity on the coordination ability of CSMA and STDMA with a CS-threshold of $-91 \mathrm{dBm}$, an RX-threshold of $-88 \mathrm{dBm}$ and a vehicle density of $85 \mathrm{veh} / \mathrm{km}$

\section{Impact of fading on the coordination ability}

In order to investigate to which degree the coordination ability of both protocols is influenced by temporary channel variations we employed a Nakagami distribution on top of the power law path loss model. Figure 6 depicts the resulting PLI without any fading, with Nakagami $m=1$, and Nakagami $m=3$ for CSMA as well as STDMA. It is apparent that the coordination ability of CSMA decreases as the severity of fading increases. This is quite intuitive, since with more severe fading the probability increases that a node is not able to declare CCA-busy and starts transmitting itself, leading to an increased PLI even in close range. For STDMA no such clear distinction can be made. Despite varying fading intensities the PLI values hardly differ, where in close range the PLI slightly increases with more severe fading and in far ranges the PLI even slightly decreases, compared to non fading conditions at all. This can be explained by the fact that fading conditions lead to an increased number of packets in close range that can not be decoded, consequently their reservation information are missed and this then leads to an increase in incoordination. As the considered range is increased this effect diminishes, as receivers which are outside of the designated communication range might still receive some packets once in a while. Since every STDMA-packet contains some additional reservation information (e.g. the next used slot within the same frame or for how long the current slot is being used) this information can then be used by the receiving station to decrease the likelihood of incoordinated transmissions.

Figure 7 depicts the resulting PDR for fading conditions with Nakagami $m=1$ and Nakagami $m=3$ for CSMA and STDMA. Compared to non fading conditions (see Figure 5) the PDR drops faster over distance, but successful packet receptions are still possible above the designated communication distance of $\approx 400$ meters. With less severe fading $(m=3)$ the PDR up to a distance of $550 \mathrm{~m}$ is only marginally better for STDMA, whereas for larger distances both protocols do not show any noticeable difference. More severe fading $(m=1)$ is less detrimental for the reservation based approach of STDMA, resulting in a difference of up to $5 \%$ in PDR, due to the reasons that were given for the PLI explanation.

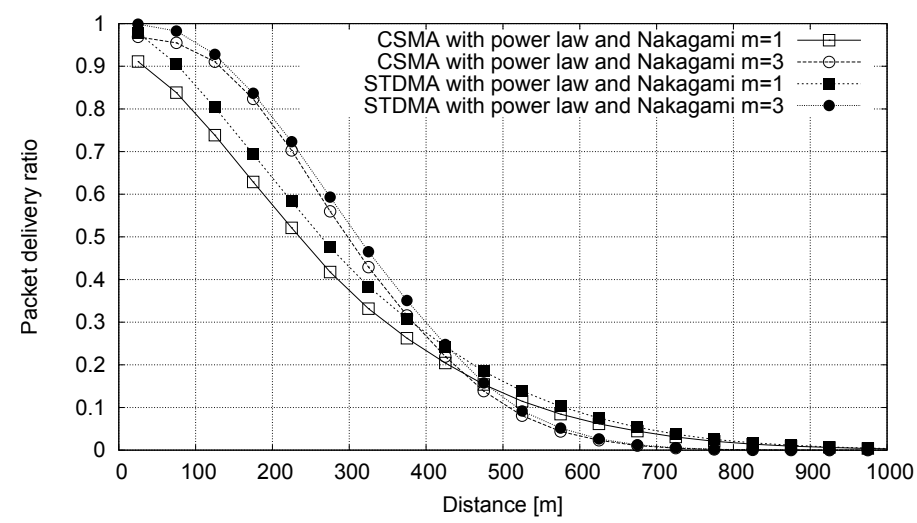

Fig. 7. Impact of fading with varying intensity on PDR when using CSMA and STDMA with a CS-threshold of $-91 \mathrm{dBm}$, an RX-threshold of $-88 \mathrm{dBm}$ and a vehicle density of $85 \mathrm{veh} / \mathrm{km}$

\section{SUMMARY AND OUTLOOK}

In this work, we have performed an analysis on how the performance of CSMA and STDMA is influenced by the existence of hidden nodes and fading conditions, as well as quantified the impact of an extended carrier sensing range. Since the overall performance is only subtly different, our focus was not to state one protocol better than the other, but to understand under which conditions one protocol performs better. In particular, our analysis shows to which degree CSMA can make use of its strength for primarily relying on the CS-range for coordinating the channel and how STDMA on the other hand is better able to cope with severe fading conditions by being able to incorporate reservation information received in the past. For future work we plan to extend this analysis to further include mobility and to consider the distribution of packet inter arrival times (indicating freshness) as another measure to assess the strengths and weaknesses of both protocols.

\section{REFERENCES}

[1] Eurocontrol. VHF Digital Mode 4. http://www.eurocontrol.int/services/ vhf-digital-mode- 4 .

[2] A. C. V. Gummalla and J. O. Limb. Wireless medium access control protocols. IEEE Communications Surveys Tutorials, 3(2):2-15, 2000.

[3] H. Hartenstein and K. Laberteaux. VANET Vehicular Applications and Inter-Networking Technologies. Intelligent Trans. Systems. Wiley, 2009.

[4] ITU. Technical Characteristics for an Automatic Identification System Using Time Division Multiple Access in the VHF Maritime Mobile Band. Technical Report ITU-R M.1371-4, 2010.

[5] K. Sjöberg, E. Uhlemann and E.G. Ström. How severe is the hidden terminal problem in VANETs when using CSMA and STDMA? In Proc. IEEE Vehicular Technology Conference, 2011.

[6] J. Kunisch and J. Pamp. Wideband car-to-car radio channel measurements and model at $5.9 \mathrm{GHz}$. In Proc. IEEE Vehicular Technology Conference, VTC 2008-Fall, Sept 2008.

[7] J. Mittag. Characterization, Avoidance and Repair of Packet Collisions in Inter-Vehicle Communication Networks. PhD thesis, Karlsruhe Inst. of Technology, 2012. https://dsn.tm.kit.edu/article.php?publication_id=248.

[8] M. Nakagami. The m-distribution - A general formula of intensity distribution of rapid fading. In W. C. Hoffmann, editor, Statistical Methods in Radio Wave Propagation. Elmsford, NY, 1960.

[9] K. Sjöberg. Medium Access Control for Vehicular Ad Hoc Networks. $\mathrm{PhD}$ thesis, Chalmers University of Technology, 2013.

[10] T. Gaugel, J. Mittag, H. Hartenstein, S. Papanastasiou, and E.G. Ström. In-depth analysis and evaluation of self-organizing TDMA. In Proc. IEEE Vehicular Networking Conference (VNC), pages 79-86, Dec 2013.

[11] F. Tobagi and L. Kleinrock. Packet switching in radio channels: Part ii-the hidden terminal problem in carrier sense multiple-access and the busy-tone solution. IEEE Transactions on Communications, 23(12):1417-1433, Dec 1975. 